\title{
Aplicaciones y ventajas estéticas de la reducción interproximal de esmalte
}

\author{
Dora Liliana Meneses Bedoya, Odont. ${ }_{1}$, Paola Botero Mariaca ${ }^{\star}$, Esp. $_{2}$ \\ Facultad de Odontología, Universidad Cooperativa de Colombia \\ ${ }_{2}$ Facultad de Odontología, Universidad Cooperativa de Colombia y Universidad CES \\ Medellín, Colombia
}

Recibido: 17 de abril de 2012. Aprobado: 6 de diciembre de 2013 .

*Autor de correspondencia: Paola Botero Mariaca, Facultad de Odontología, Universidad Cooperativa de Colombia, sede Medellín, Colombia. Calle 4 sur n. ${ }^{\circ} 43 \mathrm{~A}-26$, teléfono: 2706466, ext. 4112, correo electrónico: paola.botero@campusucc.edu.co

Cómo citar este artículo: Meneses DL, Botero P. Aplicaciones y ventajas estéticas de la reducción interproximal de esmalte. Rev Nac 0dontol. 2014;10(18):67-73. doi: http://dx.doi.org/10.16925/od.v10i18.723

Resumen. Durante los últimos años, el porcentaje de adultos que busca tratamiento ortodóncico ha ido en aumento. Con estos pacientes las demandas estéticas y las condiciones orales complican el tratamiento. El enfoque multidisciplinario con miras a realizar tratamientos más conservadores que eviten sacrificar dientes sanos es una de las metas en estos pacientes. La utilización de métodos como el desgaste dentario interproximal es una alternativa preventiva y terapéutica que, realizada con cuidado y conocimiento, no causa daño a los dientes y tejidos periodontales, facilita la corrección del apiñamiento y mejora las formas dentales y triángulos negros. Además, brinda resultados más estables, funcionales y estéticos para los pacientes.

Palabras clave: ortodoncia, periodonto, apiñamiento, desgaste.

\section{Applications and aesthetic advantages of interproximal enamel reduction}

Abstract. In recent years, the percentage of adults seeking dental treatment has been on the rise. Among these patients, aesthetic demands and oral conditions complicate treatment. A multidisciplinary approach with a view to conducting more conservative treatments that avoid sacrificing healthy teeth is one of the goals for these patients. Using methods such as interproximal tooth wear is a preventative and therapeutic alternative that, when executed with care and knowledge, does not damage teeth or periodontal tissues, helps correct crowding, and improves tooth shape and black triangles. Furthermore, it provides more stable, functional and aesthetic results for patients.

Keywords: orthodontics, periodontium, crowding, wear.

\section{Introducción}

El número de adultos que necesitan tratamiento ortodóncico está en incremento. Se estima que en los últimos años cerca del $40 \%$ de los pacientes ortodóncicos son adultos. Se presenta entonces un desafío por la demanda estética debida a sus condiciones dentales que pueden complicar el tratamiento, tales como desgastes, restauraciones mal contorneadas y enfermedad

\section{Aplicações e vantagens estéticas da redução interproximal de esmalte}

Resumo. Durante os últimos anos, a porcentagem de adultos procurando tratamento ortodôntico tem aumentado. Com esses pacientes, as demandas estéticas e as condições orais complicam o tratamento. A abordagem multidisciplinar com o objetivo de realizar tratamentos mais conservadores que evitem sacrificar dentes saudáveis é uma das metas nesses pacientes. A utilização de métodos como o desgaste dentário interproximal é uma alternativa preventiva e terapêutica que, realizada com cuidado e conhecimento, não causa danos aos dentes e tecidos periodontais, facilita a correção do apinhamento e melhora as formas dentais e triângulos negros. Além disso, fornece resultados mais estáveis, funcionais e estéticos para os pacientes.

Palavras-chave: ortodontia, periodonto, apinhamento, desgaste.

periodontal [1]. La solución a estos problemas enfatiza la estrecha relación que debe existir entre ortodoncista, periodoncista y protesista para obtener resultados que beneficien a largo plazo al paciente. Por otro lado, se ha incrementado la terapia no extraccionista dentro de los tratamientos ortodóncicos, ganando popularidad la reducción interproximal de esmalte como parte de un procedimiento conservador $[2,3]$. 
La reducción interproximal de esmalte es un procedimiento clínico que consiste en remover parte de la estructura dentaria (sólo esmalte) del área del contacto interproximal de manera controlada y moderada. El procedimiento tiene el fin de crear espacios para facilitar el alineamiento dentro del tratamiento ortodóncico y favorecer una mejor morfología o forma al diente cuando este presenta alteraciones estructurales $[4,5]$. Es una técnica que puede ser usada de manera independiente para contornear superficies dentales o como coadyuvante durante tratamientos restaurativos prostodónticos y ortodóncicos [6]. Comparado con el tratamiento de extracciones, la reducción interproximal puede reducir hasta en ocho meses el tiempo de tratamiento [7, 8] (figura 1).

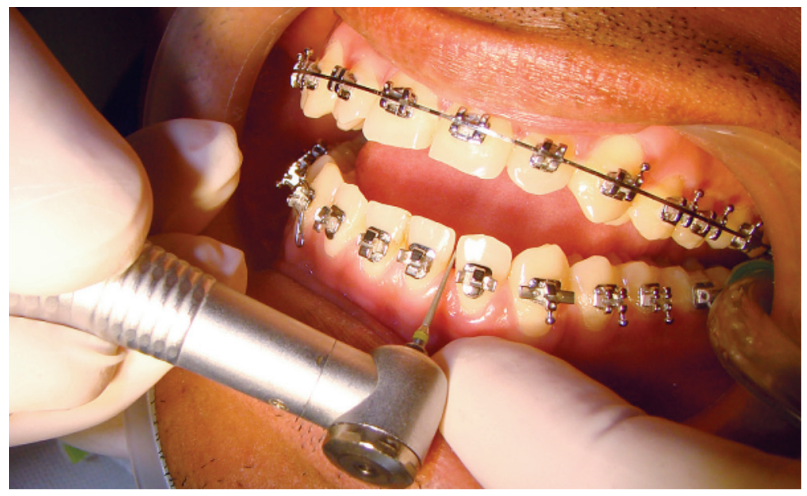

Figura 1. Procedimiento de desgaste interproximal con fresa de diamante de grano ultrafino para aliviar apiñamiento inferior Fuente: elaboración propia

La reducción interproximal es un método que ofrece varias ventajas: alivia apiñamientos leves a moderados [9], favorece mayor estabilidad de la posición dentaria al compararla con los procedimientos de expansión, reduce significativamente el tiempo de tratamiento, permite mantener las dimensiones transversales y, además, controla la inclinación de los dientes anteriores y la distancia intercanina $[1,10]$.

Al realizar una reducción interproximal no se evita la recidiva del apiñamiento en la zona anteroinferior, pero ayuda a que esta sea menor al lograr aplanar las caras proximales de los dientes transformando los puntos de contacto en áreas más anchas que dificultan la recidiva $[11,12]$.

Sin contraindicación específica no debe realizarse en pacientes con gingivitis generalizada, enfermedad periodontal o higiene oral deficiente, ya que podría causar daño o deterioro a los tejidos e incrementar el riesgo a descalcificaciones y caries [13, 14].

\section{Evolución histórica}

Una de las referencias más antiguas del desgaste interproximal es Linn en 1943, que reportó el tratamiento exitoso de un caso con discrepancia dentaria utilizando esta técnica. Fue realizada al inicio del tratamiento ortodóncico, previa a la colocación del sistema multibandas [15]. Al año siguiente, Ballard lo recomendó para el segmento anterior cuando faltaba balance en esta zona [16], mientras que Begg, en 1954, propone que la base teórica de la utilización del desgaste interproximal surge a partir de las observaciones de la dentición de aborígenes australianos, en los que encontró desgastes no sólo en las superficies oclusales de los dientes, sino también en las interproximales. Estos desgastes se explican por un mecanismo de abrasión dentaria natural generada por los hábitos alimenticios [17]. En 1958, Bolton presentó su estudio "Desarmonía en el tamaño de los dientes y su relación con el tratamiento de la maloclusión", en el cual soporta la necesidad de usar el degaste interproximal en problemas de discrepancia de arcos [18]. En 1972, Peck y Peck demostraron que existe una relación directa entre la forma de los incisivos mandibulares y la presencia o no de apiñamiento; además, describieron el denominado índice mesiodistal y bucolingual como una expresión numérica de la forma de los incisivos inferiores observados desde incisal. El estándar recomendado para realizar desgaste interproximal para los incisivos centrales inferiores es un índice $\mathrm{MD} / \mathrm{BL}$ de $88-92 \%$, y la de los incisivos laterales de $90-95 \%$ [19].

Por otro lado, Kelsten recomendó el uso de procedimientos mecánicos para el desgaste y la realización del alineamiento de los dientes previo al desgaste [20]. A partir de allí, se consolida como una alternativa de tratamiento y se perfeccionan los elementos empleados para hacerla.

\section{Descripción de la técnica}

En 1985 y 1987, Sheridan propone y protocoliza una técnica que se fundamenta en los procesos naturales de abrasión interdental y se realiza con una pieza de mano de alta velocidad, principalmente en zonas posteriores distal a los caninos y mesial de los segundos molares 
$[8,21]$. La cantidad de desgaste está directamente relacionada con la cantidad de apiñamiento por resolver y sus indicaciones señalan remover solamente $1 \mathrm{~mm}(0,5$ por superficie proximal), ya que los dientes posteriores son los que presentan mayor grosor de esmalte. Se recomienda abrir espacios previos al solo tallado con un resorte abierto o elastómero, para favorecer un mejor acceso y control visual [22]. También se deben corregir previamente las rotaciones dentarias para favorecer una mejor morfología al tallar. Con el fin de proteger la encía, se recomienda utilizar un alambre indicador (0,20 de cobre) ubicado gingival al punto de contacto y realizar la reducción en un sitio a la vez, iniciando de posterior a anterior. Se inicia con fresas de carburo y luego de diamante muy finas, de punta redondeada para evitar escalones durante el desgaste, la cual se pincela desde cervical a oclusal bajo irrigación abundante
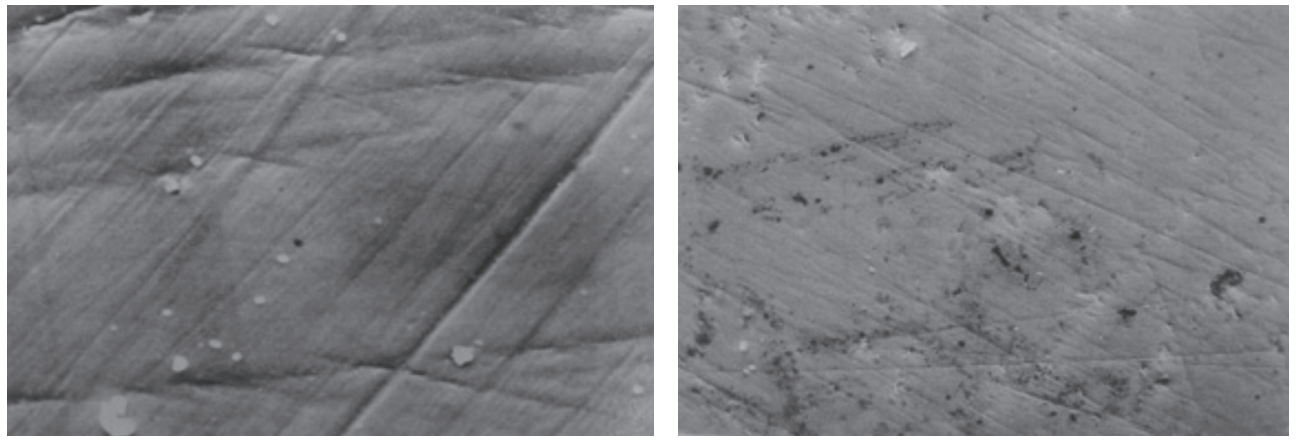

Figura 2. Superficie pulida con discos Sof-Lex

${ }^{*}$ Se observan ranuras muy leves alternadas con zonas bien pulidas

Fuente: [24]

Otro instrumento que puede emplearse para el desgaste es una pieza a la que se le incorporan lijas metálicas, la cual tiene mejor rendimiento al obtener mayor desgaste que las lijas manuales [6].

\section{Consecuencias del desgaste}

A partir de 1956, surgió la necesidad de realizar estudios que evaluaran el efecto del desgaste en la estructura del diente. Inicialmente se evaluó si el desgaste interproximal realizado con lijas incrementaba el riesgo de caries dental en los dientes antero-inferiores y se encontró que se producían ranuras en el esmalte que generaban mayor atrapamiento de placa bacteriana, lo que podría incrementar la susceptibilidad a la caries.
[23]. Se debe buscar finalizar las superficies proximales con adecuada morfología y textura, para lo cual recomienda fresas de diamante de grano extrafino y discos Sof-Lex posterior al desgaste. El paciente debe realizarse enjuagues de flúor para favorecer el potencial de remineralización en los dientes desgastados [9].

A pesar de utilizar los métodos de pulido, después de realizado el desgaste, no se consiguen las mismas características morfológicas del esmalte inicial. Sin embargo, las superficies más lisas y con menos retenciones para la placa son conseguidas con discos de pulido Sof-Lex usados, luego de la reducción con las fresas de carburo [24] (figura 2). Recientes estudios demuestran que el uso de discos de diamante perforados refrigerados con menos de $30 \mu \mathrm{m}$, de tamaño de grano, y la subsecuente pulida con discos Sof-Lex generan superficies tan o más suaves que el esmalte no tratado [25].
Se genera entonces la necesidad de realizar estudios que valoren la forma dental y la conveniencia del desgaste, teniendo en cuenta el conocimiento de las características dentales como formas y grosor del esmalte, características fundamentales para llevar a cabo una técnica con menores problemas.

En 1993, Shillingbourg, con su artículo "Grosor del esmalte y la dentina”, sirvió como base científica para trabajar sobre la cantidad de esmalte que podría ser segura y removida de manera precisa en cada superficie dental [26]. Otros estudios demuestran que una adecuada técnica no resulta en un incremento en caries en los pacientes sometidos a desgaste [27]. Los hallazgos histológicos muestran que la remodelación dental es posible con menores reacciones pulpares y dentinales y, por tanto, menores reacciones clínicas y radiográfi- 
cas. Las ranuras creadas por el desgaste (con profundidad entre 15 y 30 micras) pueden ser visibles hasta 1 año después del procedimiento, pero no son predisponentes para la caries [28-30].

Seguimientos por más de 10 años luego del desgaste interproximal muestran que no hay indicación de que el recontorneado de los dientes incrementa el riesgo al desarrollo de futuras caries, daños a los tejidos duros y blandos periodontales o conduzca a un incremento en la sensibilidad a los cambios de temperatura [31].

El grosor del esmalte disponible para la reducción ha sido evaluado a través de radiografías coronales tomadas en sujetos entre los 20 y 35 años de edad, en los que se mostró que no existen diferencias significativas en cuanto a sexo; sin embargo, en el ancho del esmalte mesial y distal sí se presentan diferencias según el tipo de diente. El esmalte de los segundos molares es significativamente más grueso $(0,3$ a $0,4 \mathrm{~mm})$ que el de los premolares. Así mismo, el esmalte distal es más grueso que el mesial [32]; en promedio, 1,29 $\mathrm{mm}$ en las caras distales cuando se compara con las superficies mesiales $1,13 \mathrm{~mm}$ [33] (figura 3).

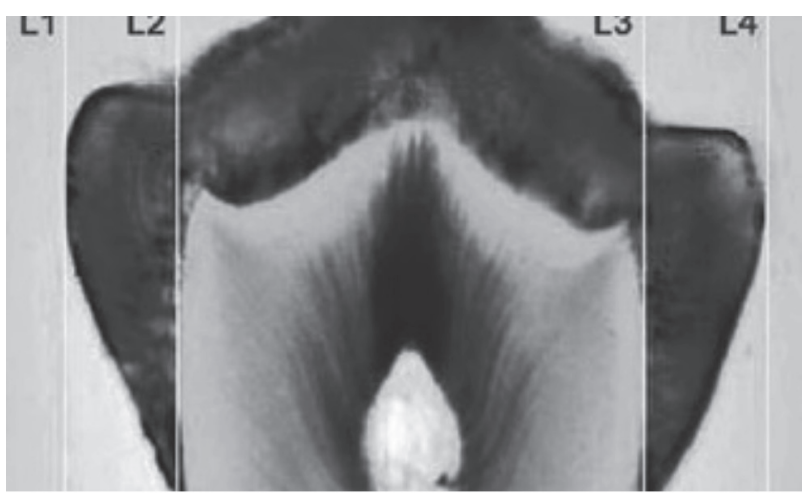

Figura 3. Extensión del esmalte proximal mesial y distal Fuente: [33]

Existen aproximadamente $10 \mathrm{~mm}$ de esmalte en los dientes postero-inferiores desde la cara mesial del primer premolar hasta la cara distal del segundo molar en cada lado. Asumiendo que se puede reducir el $50 \%$ de esmalte, los premolares y molares de ambos lados pueden brindar hasta 9,8 $\mathrm{mm}$ de espacio adicional para el realineamiento de los dientes mandibulares [32]. A partir de estos estudios, se sugiere un desgaste de $0,2 \mathrm{~mm}$ para los incisivos centrales y $0,25 \mathrm{~mm}$ para los incisivos laterales, e incluso hasta $0,3 \mathrm{~mm}$. Para caninos se recomienda $0,4 \mathrm{~mm}$ en cada superficie. Por otro lado, Alexander permite sólo 0,25 mm para todos los dientes y Sheridan
$0,8 \mathrm{~mm}$ por cada superficie de los posteriores y 0,25 en los dientes anteriores; otros aseguran que con una reducción del 50\% del esmalte original es aceptable [21, 34]. Sin embargo, no existe consenso en cuanto a la cantidad de esmalte que se debe retirar. Aunque el tamaño dental no guarda proporción con el grosor del esmalte, es más aconsejable la reducción interproximal en los dientes de mayor tamaño que en dientes pequeños [35].

La forma y el tamaño de los dientes se puede clasificar en tres tipos: cuadrada, ovoidea y triangular [36]. La interrelación de los dientes depende de su forma: los de forma cuadrada tienen mayor superficie de contacto entre ellos, más cercana al borde gingival y una distancia interradicular disminuida; los de forma triangular poseen una menor superficie de contacto cercana al borde incisal y presentan una mayor distancia interradicular; y finalmente, los de forma ovoidea están en un punto intermedio. Con base en estas características, los dientes con la mejor forma para realizar el desgaste interproximal son los triangulares, ya que permiten un recontorneado de su superficie sin producir un acercamiento excesivo de las raíces dentarias ni compresión de la papila interdental (figuras 4 y 5) [35].
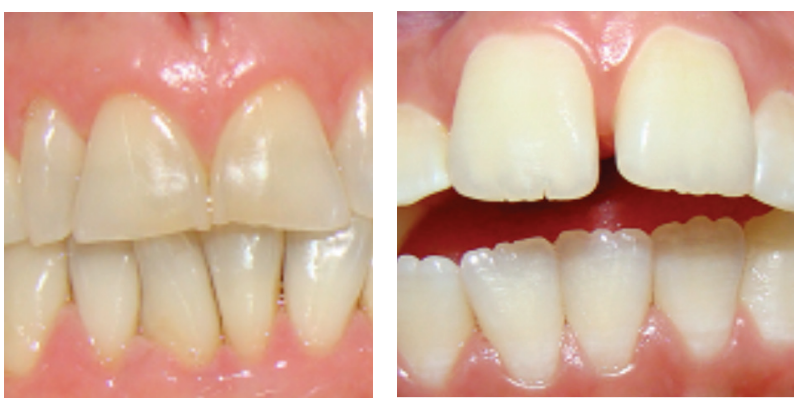

Figura 4. Forma de dientes. En la primera imagen se observan unos dientes triangulares y en la segunda, unos dientes cuadrados Fuente: elaboración propia
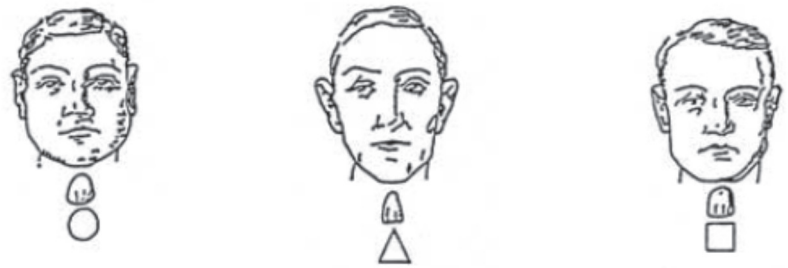

Figura 5. La forma de los dientes tiene relación con la forma de la cara

Fuente: [36] 


\section{Implicaciones estéticas y biológicas en el ámbito dental y periodontal}

Con la reducción de esmalte y recontorneado cosmético se pueden lograr mejores formas dentales y mantener a largo plazo el alineamiento de los incisivos mandibulares; además, se logra la eliminación de triángulos negros presentes en pacientes con dientes triangulares con punto de contacto incisal [37]. El recontorneado puede ser requerido cuando los caninos son usados para reemplazar los incisivos laterales genéticamente ausentes o extraídos [38]. Según Zachrisson, el objetivo del desgaste es recontornear la forma anormal de los dientes hacia una morfología más ideal y los candidatos más opcionados son los dientes triangulares o premolares ovales [37].

Es importante tener en consideración que hay limitantes para la reducción del esmalte como su mor- fología, grosor del esmalte, convexidad de la superficie proximal [39-41]. Asimismo, es importante considerar no solo las formas del diente, sino la distancia entre la cresta ósea y el punto de contacto. Una distancia igual o menor a $5 \mathrm{~mm}$ favorece que la papila llene completamente el 100\% del nicho gingival: si es de $6 \mathrm{~mm}$, la papila es hallada en $56 \%$ de los casos y si es de $7 \mathrm{~mm}$ o más, se presenta sólo en $27 \%$ de estos.

A nivel periodontal, las ventajas de la reducción interproximal son la prevención o reducción de retracciones de papilas, además del desarrollo de nichos gingivales abiertos y la provisión de adecuados conectores en la región incisiva. Todo lo anterior facilita el óptimo llenado de la papila gingival, lo cual genera mejor apariencia estética y evita problemas periodontales por retención crónica de alimentos, tema de especial importancia cuando se tratan pacientes adultos [42] (figura 6).
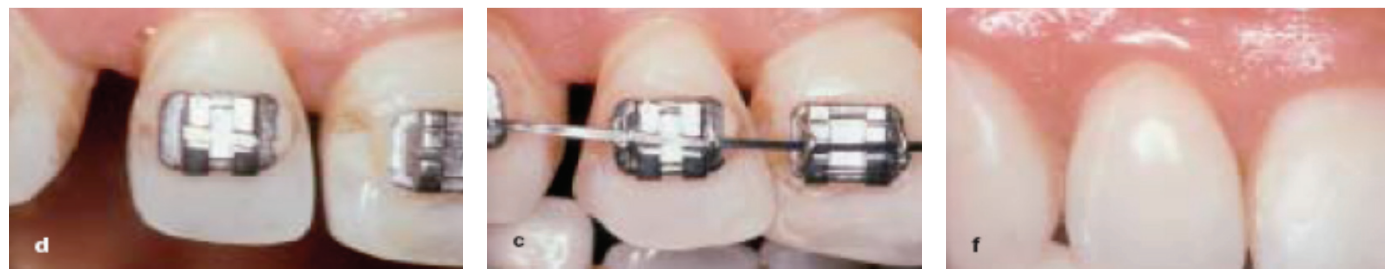

Figura 6. Eliminación de triángulos negros y llenado papilar con la ortodoncia y el desgaste interproximal Fuente: [42]

La recolocación del punto de contacto más apical y el aumento del área de los conectores también es lograda con el desgaste. Los conectores, diferentes al punto de contacto, se definen como las áreas donde los dientes anteriores se unen y son generalmente áreas más amplias (aproximadamente $2 \times 2 \mathrm{~mm}$ ) que ayudan a la estética de la región anterior. La regla 50-40-30 define que la zona de conexión ideal entre los incisivos maxilares centrales es en el 50\% de la longitud de estos. La zona de conexión ideal entre un incisivo lateral superior y un incisivo central sería de $40 \%$ de la longitud del incisivo central, mientras la zona de conexión óptima entre un canino superior y el incisivo lateral cuando se ve en vista lateral es $30 \%$ de la longitud del incisivo central [43]. Teniendo presente que el área del conector debe ir paralela a la línea mediofacial para favorecer la salud periodontal y la estética, esta se puede mejorar con una reducción interproximal en los casos en que la ortodoncia fuera insuficiente [35].
Una de las críticas al desgaste interproximal es que este genera una proximidad radicular que genera futuros problemas periodontales; sin embargo, Zachrisson, en el 2007, concluyó que la distancia horizontal entre las raíces de los incisivos fue la misma o más grande que la correspondiente distancia en sujetos que no recibieron desgaste interproximal [39].

Se considera proximidad radicular cuando existe $0,8 \mathrm{~mm}$ o menos de hueso o tejido interdental entre dos raíces adyacentes. La proximidad debe ser medida en radiografías periapicales y se han descrito diferentes grados: severidad $1(0,5-0,8 \mathrm{~mm}$ de hueso esponjoso interradicular), severidad $2(0,3-0,5 \mathrm{~mm}$ de hueso cortical y tejido conectivo de unión) y severidad 3 (menos de $0,3 \mathrm{~mm}$ de sólo tejido conectivo de unión) [44]. En pacientes con enfermedad periodontal, se cree que la proximidad radicular es un factor de riesgo para la progresión de dicha enfermedad, es decir, que es asociada con una mayor probabilidad de enfermedad, pero no 
necesariamente un factor causal. Además, el tratamiento periodontal (detartraje, alisado radicular) y el acceso quirúrgico pueden verse limitados en dichos casos. Sin embargo, en un estudio que evaluó las diferencias periodontales de dientes con espacios normales entre sus raíces y dientes con proximidad radicular y un hueso septal delgado, se concluyó que aparentemente la cantidad de hueso entre las raíces no influye directamente en la progresión de la enfermedad periodontal [45, 46]. Esto se debe a que distancias interradiculares de $0,3 \mathrm{~mm}$ a $0,5 \mathrm{~mm}$ pueden tener un ligamento periodontal normal aun en la ausencia de hueso. Es aparente, desde la revisión de la literatura, que después de una cuidadosa remoción del esmalte interproximal, los resultados a largo plazo pueden ser denticiones saludables con resultados periodontales y dentales sin efectos negativos [47-50].

\section{Conclusiones}

1. Al evaluar las ventajas, consideraciones e indicaciones del desgaste interproximal, puede deducirse que, aunque parece una técnica sencilla, requiere diagnóstico riguroso del caso utilizando todas las ayudas para evaluar discrepancia dentaria o apiñamiento, diagnóstico radiográfico para evaluar posición radicular, conocer convexidad, superficies proximales, ancho del esmalte y valorar la salud periodontal.

2. El manejo interdisciplinario es imprescindible y sugiere el acompañamiento del rehabilitador, que es el que conoce y maneja de manera más precisa formas, contornos, translucidez, proporciones y color de los dientes, y del periodoncista, que nos ayuda a evaluar y a considerar el manejo periodontal óptimo en pacientes ya comprometidos.

3. No hay evidencia de que la reducción del esmalte interproximal, dentro de los límites reconocidos y en apropiadas situaciones, pueda causar daño al diente, tejido gingival, adherencia epitelial o nivel de hueso alveolar.

\section{Referencias}

[1] Kurth JR, Kokich VG. Open gingival embrasures after orthodontic treatment in adults: Prevalence and etiology. Am J Orthod Dentofacial Orthop. 2001;120(2):116-23.
[2] O'Connor BM. Contemporary trends in orthodontic practice: a national survey. Am J Orthod Dentofacial Orthop. 1993;103(2):163-70.

[3] Arman A, Cehreli SB, Ozel E, Arhun N, Cetinsahin A, Soyman M. Qualitative and quantitative evaluation of enamel after various stripping methods. Am J Orthod Dentofacial Orthop. 2006;130(2):131.e7-14.

[4] Pinheiro ML. Interproximal enamel reduction. World J Orthod. 2002;3:223-32.

[5] Tuverson DL. Anterior interocclusal relations. Part 1. Am J Orthod. 1980;78(4):361-70.

[6] Sheridan JJ. On air-rotor stripping. J Clin Orthod. 2008;42(7):381-8.

[7] Germec D, Taner TU. Effects of extraction and nonextraction therapy with air-rotor stripping on facial esthetics in postadolescent borderline patients. Am J Orthod Dentofacial Orthop. 2008;133(4):539-49.

[8] Rossouw PE, Tortorella A. A pilot investigation of enamel reduction procedures. J Can Dent Assoc. 2003;69(6):384-8.

[9] Chudasama D, Sheridan JJ. Overview. Guidelines for contemporary air-rotor stripping. J Clin Orthod. 2007;41(6):315-20.

[10] Germec-Cakan D, Taner TU, Akan S. Arch-width and perimeter changes in patients with borderline Class I malocclusion treated with extractions or without extractions with air-rotor stripping. Am J Orthod Dentofacial Orthop. 2010;137(6):734.e1-7.

[11] Boese LR. Fiberotomy and reproximation without lower retention, nine years in retrospect: part I. Angle Orthod. 1980;50(3):88-97.

[12] Taner TU, Haydar B, Kavuklu I, Korkmaz A. Short-term effects of fiberotomy on relapse of anterior crowding. Am J Orthod Dentofacial Orthop. 2000;118(6):617-23.

[13] Sheridan JJ. Air-rotor stripping. J Clin Orthod. 1987;21:781-8.

[14] Harfin J. Tratamiento ortodóncico en el adulto. Buenos Aires: Panamericana; 1999.

[15] Linn J. Paper Read before southern section. Los Angeles: Pacific Coast Society of Orthodontists; 1943.

[16] Ballard ML. Asymmetry in tooth size: A factor in the etiology, diagnosis, and treatment of malocclusion. Angle Orthod. 1944;14(3):67-71.

[17] Begg PR. Stone Age man's dentition. Am J Orthod.1954;40:298-312.

[18] Bolton W. Disharmony in tooth size and its relation to the analysis and treatment of malocclusion. Angle Orthod. 1958;28(3):113-30. 
[19] Peck H, Peck S. An index for assessing tooth shape deviations as applied to the mandibular incisors. Am J Orthod. 1972;61(4):384-401.

[20] Kelsten LB. A technique for realignment and stripping of crowded lower incisors. J Pract Orthod. 1969;3(2):824.

[21] Sheridan JJ. Air-rotor stripping. J Clin Orthod. 1985;19(1):43-59.

[22] Sheridan JJ. Air-rotor stripping update. J Clin Orthod. 1987;21(11):781-8.

[23] Sheridan JJ. The physiologic rationale for air-rotor stripping. J Clin Orthod. 1997;31(9):609-12.

[24] Piacentini C, Sfondrini G. A scanning electron microscopy comparison of enamel polishing methods after air-rotor stripping. Am J Orthod Dentofacial Orthop. 1996;109(1):57-63.

[25] Zhong M, Jost-Brinkmann PG, Radlanski RJ, Miethke RR. SEM evaluation of a new technique for interdental stripping. J Clin Orthod. 1999;33(5):286-92.

[26] Shillingburg HT Jr., Grace CS. Thickness of enamel and dentin. J South Calif Dent Assoc. 1993;41(1):33-6.

[27] Jarjoura K, Gagnon G, Nieberg L. Caries risk after interproximal enamel reduction. Am J Orthod Dentofacial Orthop. 2006;130(1):26-30.

[28] Thordarson A, Zachrisson BU, Mjör IA. Remodeling of canines to the shape of lateral incisors by grinding: a long-term clinical and radiographic evaluation. Am J Orthod Dentofacial Orthop. 1991;100(2):123-32.

[29] El-Mangoury NH, Moussa MM, Mostafa YA, Girgis AS. In-vivo remineralization after air-rotor stripping. J Clin Orthod. 1991;25(2):75-8.

[30] Radlanski RJ, Jager A, Zimmer B. Morphology of interdentally stripped enamel one year after treatment. J Clin Orthod. 1989;23(11):748-50.

[31] Zachrisson BU. Actual damage to teeth and periodontal tissues with mesiodistal enamel reduction ("stripping"). World J Orthod. 2004;5(2):178-83.

[32] Stroud JL, English J, Buschang PH. Enamel thickness of the posterior dentition: its implications for nonextraction treatment. Angle Orthod. 1998; 68(2):141-6.

[33] De Carvalho A, Vellini-Ferreira F, Scavone-Junior H, Ferreira RI. Mesiodistal width and proximal enamel thickness of maxillary first bicuspids. Braz Oral Res. 2010;24(1):58-63.

[34] Danesh G, Hellak A, Lippold C, Ziebura T, Schafer E. Enamel surfaces following interproximal reduction with different methods. Angle Orthod. 2007;77(6):1004-10.

[35] Zachrisson BU. Interdental papilla reconstruction in adult orthodontics. World J Orthod. 2004;5(1):67-73.
[36] Wiliams J. The tooth shaped. Dental Digest. 1925;31:23943.

[37] Tarnow DP, Magner AW, Fletcher P. The effect of the distance from the contact point to the crest of bone on the presence or absence of the interproximal dental papilla. J Periodontol. 1992;63(12):995-6.

[38] Zachrisson BU, Mjör IA. Remodeling of teeth by grinding. Am J Orthod.1975;68(5):545-53.

[39] Zachrisson BU. JCO/interviews Dr. Bjorn U. Zachrisson on excellence in finishing. Part 2. J Clin Orthod. 1986;20(7):536-556.

[40] Philippe J. A method of enamel reduction for correction of adult arch-length discrepancy. J Clin Orthod. 1991;25(8):484-9.

[41] Zachrisson BU. Buccal uprighting of canines and premolars for improved smile esthetics and stability. World J Orthod. 2006;7(4):406-12.

[42] Zachrisson BU, Minster L, Ogaard B, Birkhed D. Dental health assessed after interproximal enamel reduction: caries risk in posterior teeth. Am J Orthod Dentofacial Orthop. 2011;139(1):90-8.

[43] Morley J, Eubank J. Macroesthetic elements of smile design. J Am Dent Assoc. 2001;132(1):39-45.

[44] Vermylen K, De Quincey GN, Van't Hof MA, Wolffe GN, Renggli HH. Classification, reproducibility and prevalence of root proximity in periodontal patients. J Clin Periodontol. 2005;32(3):254-9.

[45] Artun J, Osterberg SK, Kokich VG. Long-term effect of thin interdental alveolar bone on periodontal health after orthodontic treatment. J Periodontol. 1986;57(6):341-6.

[46] Zachrisson BU, Nyøygard L, Mobarak K. Dental health assessed more than 10 years after interproximal enamel reduction of mandibular anterior teeth. Am J Orthod Dentofacial Orthop. 2007;131(2):162-9.

[47] Brudevold F, Tehrani A, Bakhos Y. Intraoral mineralization of abraded dental enamel. J Dent Res. 1982;65(3):456-9.

[48] Thordarson A, Zachrisson B, Mjör I. Remodeling of canines to the shape of lateral incisors by grinding: a long-term clinical and radiographic evaluation. Am J Orthod Dentofac Orthop. 1991;100:123-32.

[49] Harfin JF. Interproximal stripping for the treatment of adult crowding. J Clin Orthod. 2000;34(7):424-33.

[50] Zhong M, Jost-Brinkmann PG, Zellmann M, Zellmann $S$, Radlanski RJ. Clinical evaluation of a new technique for interdental enamel reduction. J Orofac Orthop. 2000;61(6):432-9. 